\title{
Étude comparée de la végétation de 1964 et 2011 en milieu pâturé: Cas du CRZ de Dahra
}

\author{
Ousmane Ndiaye 1,3, Amadou Tamsir Diop 2, Mamadou Diène 1, Léonard Elie Akpo ${ }^{3}$ \\ 1 Centre de Recherches Zootechniques de Dahra, Institut Sénégalais de Recherches Agricoles, Dahra, Sénégal \\ 2 Laboratoire National d'Elevage et de Recherche Vétérinaire (LNERV), Institut Sénégalais de Recherches \\ Agricoles, Dakar, Sénégal \\ 3 Laboratoire d'Écologie Végétale et d'Écohydrologie FST/UCAD, BP 5005, Dakar, Sénégal \\ Addressee mail: fisco42000@yahoo.fr
}

Original submitted in on $18^{\text {th }}$ March 2015. Published online at www.m.elewa.org on $30^{\text {th }}$ April 2015 http://dx.doi.org/10.4314/jab.v88i1.8

\section{RÉSUMÉ}

Objectif : L'étude comparative de la végétation du CRZ de Dahra en 1964 et 2011, a pour but de voir l'évolution de la phytomasse herbacée et de certains paramètres structuraux du couvert herbacé et ligneux.

Méthodologie et résultats : Avec l'aide du SIG, 55 relevés de végétation ont pu être effectués en 2011 sur les mêmes points que ceux réalisés par Raynal (1964). La richesse, la fréquence et la contribution spécifique de la flore herbacée et ligneuse sont évaluées pour ces deux années de même que l'indice de Shannon, la régularité, l'indice de Jaccard et la phytomasse herbacée. Cette dernière est soumise au test de Tukey au seuil de $5 \%$. Les résultats indiquent une baisse de la phytomasse herbacée (significative au seuil de 5\%) et de la diversité floristique. Cette dernière est marquée par une diminution de la richesse spécifique de la flore herbacée comme ligneuse. Au niveau du couvert herbacé, il est noté une augmentation de la contribution spécifique (CSi) des graminées (Poacées) tandis que celle des légumineuses (Fabacées) et des autres herbacées (phorbes) a connu une régression. Le couvert ligneux est caractérisé par une augmentation des espèces de la famille des Mimosacées et une diminution de celles des Combrétacées. Toutefois, les Mimosacées, Balanitacées et Combrétacées présentent les fréquences relatives et les contributions les plus importantes pour ces deux années.

Conclusion et application des résultats : cette étude a permis de constater une modification de la végétation du CRZ de Dahra marquée sur le plan structural par une baisse de sa richesse et de sa diversité floristique ; de même que sa phytomasse herbacée. Ces résultats peuvent, contribuer à la compréhension de l'évolution de la végétation des écosystèmes sahéliens pastoraux et, être valorisés en aménagement pastoral et agrosylvopastoral en vue d'une meilleure préservation et gestion des ressources végétales.

Mots clés : richesse, diversité, phytomasse, végétation 
Comparative study of the vegetation in a grazed area between 1964 and 2011. The case of the CRZ Dahra

\section{ABSTRACT}

Objective: A comparative study of the vegetation of the CRZ-Dahra during years 1964 and 2011, aims to appreciate the evolution of herbaceous phytomass, as well as some structural parameters of herbaceous and woody cover.

Methodology and results: Using Geographic Information System (GIS), 55 vegetation surveys were carried out in 2011 on the same points used by Raynal (1964). The species richness, the frequency and the specific contribution of herbaceous and woody plants were assessed for each year, as well as the Shannon index, the regularity, the Jaccard index and the herbaceous biomass production. The latter characteristic was subjected to an analysis of variance followed by a separation of means using the Turkey's multiple comparison procedure (with a level significance fixed at the $5 \%$ level). The results indicate a decrease in herbaceous phytomass production $(P<0,05)$ and of plant diversity. The decline in plant diversity is reflected by a decrease of species richness of both herbaceous and woody plants. In the herbaceous layer, it is noted an increase of the specific contribution (CSi) of grasses (Poaceae) while that of legumes (Fabaceae) and other herbaceous plants (Phorbs) experienced a decline. The tree cover is characterized by an increase in species of the family of Mimosaceae and a decrease of those belonging to the Combretaceae. However, Mimosaceae, Balanitacea and Combretaceae presented the most significant relative frequencies and contributions for the two years of study. Conclusion and application of the results: This study revealed a vegetation change at the CRZ Dahra. This shift is structurally marked by a decline of its floristic richness and diversity, as well as of its herbaceous phytomass production. These results may contribute to a better understanding of the evolution of the vegetation in Sahelian pastoral ecosystem. They may also be valorized for the planning of agrosylvopastoral areas in order to improve the preservation and the management of plant resources.

Keywords: richness, diversity, phytomass, vegetation

\section{INTRODUCTION}

Le Ferlo, comme la plupart des zones semi-arides de l'Afrique occidentale, est affecté par les séries de sécheresses des années 70 et 80 et par la forte variabilité spatio-temporelle des précipitations et de leur distribution. Ces manifestations ont, à elles seules, ou combinées à d'autres facteurs (zooanthropiques), engendrées d'importantes fluctuations de la composition botanique et de la production végétale (Breman et De Ridder, 1991) et conditionnent ainsi la dynamique des parcours sahéliens (Behnke et Scoones, 1993 ; Ellis et al., 1993). Ces derniers, qui sont des écosystèmes naturels où coexistent une végétation herbacée continue et une strate ligneuse plus ou moins discontinue (Akpo et al., 2000), jouent un rôle économique important sur le plan agro-sylvopastoral. Ils offrent des biens et services aux populations locales à travers la cueillette, l'élevage, la pharmacopée et la disponibilité de matériaux de construction, assurant ainsi leur subsistance (Scoones, 1999). Ces pâturages naturels contribuent également au maintien de la biodiversité (Briemle et al. 1997 ; Zdanowicz et al. 2005) et de la fertilité des sols (Cossée, 1999), à la protection des terres contre l'érosion (Briemle et Elsässer 1997), à la régulation du climat à travers la séquestration du carbone dans les sols (Soussana et al., 2007; 2010). Ils constituent, à travers sa composante herbacée, la base de l'alimentation des ruminants domestiques (Grouzis, 1988 ; Grouzis et al, 1991 ; Akpo et al, 2003, Diop, 2007, Akpo ; 1990, 1992 ; Ickowicz, 1995). En mi-saison sèche, la composante ligneuse prend le relais pour assurer la fourniture en azote et en protéine aux animaux (Baumer, 1992 ; Guinko et al., 1989 ; Guinko, S., 1991 ; Ickowicz et al., 2005 ; Von Maydell, 1983) favorisant ainsi leur maintien et leur entretien pendant cette période. Ces écosystèmes naturels, sous l'effet combiné de la sécheresse, de la forte variabilité de la pluviosité et de la pression d'exploitation, connaissent une dégradation accrue qui se manifeste par une modification de sa structure (distribution horizontale, 
verticale, composition floristique, richesse et diversité spécifique de la végétation), de son fonctionnement (phytomasse) et des systèmes de production, accentuant ainsi la vulnérabilité des

\section{MATÉRIEL ET MÉTHODES}

Présentation de la zone d'étude : Le Centre de Dahra, implanté depuis 1950, est situé approximativement à $15^{\circ} 21$ de latitude Nord et à $15^{\circ} 28$ longitude Ouest. II est délimité au Nord par la commune de Kamb et au Sud par celle de Thiamène et fait parti du département de Linguère (figure 13), donc en plein cœur du Sénégal dans la zone sylvo-pastorale. Cette région, essentielle pour l'élevage sénégalais, se situe à la limite méridionale du populations locales. D'où la nécessité de cette étude qui se veut de comparer la végétation du CRZ de Dahra entre deux période 1964 et 2011 afin de faire un état des lieux de ses ressources végétales.

domaine sahélien, le secteur sahélo-soudanien. Le CRZ de Dahra forme une concession de 6800 ha divisée en deux parties juxtaposées (figure 1). La petite concession de 900 ha abrite tous les bâtiments techniques, administratifs et les logements du personnel. La grande concession de 5900 ha constituait jusqu'au début des années 90 la zone de pâturages (extensifs) comprenant 19 parcelles (Sow et al., 1988).

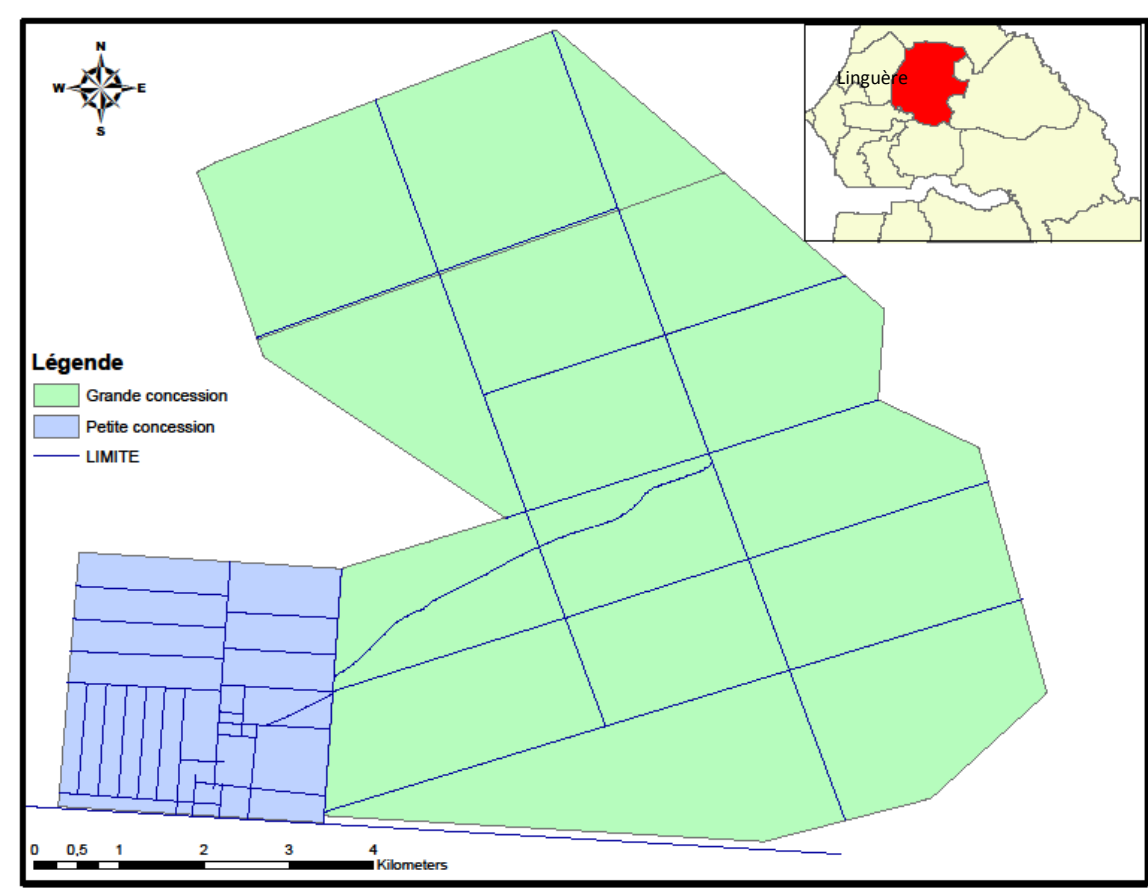

Figure 1 : localisation et plan du CRZ de Dahra

Climat, sols, végétation et systèmes de production Le climat : La pluviométrie varie d'une année à l'autre avec une moyenne de $371,67 \mathrm{~mm}$ au cours des cinquante dernières années (données pluviométriques de la station météo du "Centre de recherches zootechniques de Dahra " (Ndiaye et al., 2014). L'harmattan est dominant, mais la mousson, vent chaud et humide est activé pendant la saison des pluies. La température reste élevée dans la plupart de l'année. Les plus basses se produisent entre Décembre et Février et le plus élevées entre Avril, Mai et Juin; la moyenne est estimée à environ $30^{\circ} \mathrm{C}$ (Ndione et al, 2013).

Les sols : Cinq unités pédologiques ont été distinguées par Audry (1962) au niveau du centre, il s'agit des sols diors localisés dans la partie nord, centre et sud-est; des sols brun-rouges situés à l'est, au nord-est et au sud-est; du complexe ferrugineux-calcimorphe des zones sableuses à calottes calcaires, dans la partie centre, sud et sud-ouest ; des sols ferrugineux tropicaux à drainage moyen ou médiocre, localisés essentiellement au sud et sud-ouest et ; du complexe mal drainé des bas-fonds et 
mares temporaires, plus présent dans la partie sud, sudouest et centre.

La végétation: La végétation comprend un couvert herbacé et ligneux. Le couvert herbacé est constitué d'espèces annuelles dominées par des graminées dont Cenchrus bifrorus, Zornia glochidiata, Schoenefeldia gracilis, Dactyloctenium aegyptium, Aristida mutabilis (Fensholt, 2003, Ndiaye et al, 2014). La flore ligneuse est composée d'arbres et d'arbustes dominés par Balanites aegyptiaca, Boscia senegalensis, Acacia Sénégal, Acacia seyal, Acacia radiana, Pterocarpus lucens, Combretum glutinosum, Combretum aculeatum (Diallo, 1996).

Les systèmes de production : L'élevage extensif est le système dominant dans la zone d'étude. Ce mode d'élevage extensif traditionnel coexiste plus en plus d'un système de semi-intensive qui est basé sur la complémentation des animaux avec des aliments concentrés principalement dans la production laitière
(Ndione et al, 2013). Il est également noté la production de la gomme arabique avec la société Asiyla Gum company.

Collecte de données : Au niveau du CRZ de Dahra, 55 sites ont fait l'objet en 1964 de relevés phytosociologiques réalisés par Raynal et qui a permis une caractérisation de sa végétation sur le plan structural et fonctionnel. Avec l'aide du SIG, des relevés de végétation ont pu être effectués sur les mêmes sites que ce dernier en 2011 (figure 2). Pour l'évolution de la structure floristique du Centre en 1964 et 2011, une comparaison de la richesse floristique et du recouvrement des espèces par la méthode Braun-Blanquet est faite. Par rapport au recouvrement qui est un paramètre semi-quantitatif, il a été transformé en recouvrement moyen selon la méthode de Dufrêne (2003), qui utilise la médiane pour chaque classe de recouvrement donnée par Braun-Blanquet.

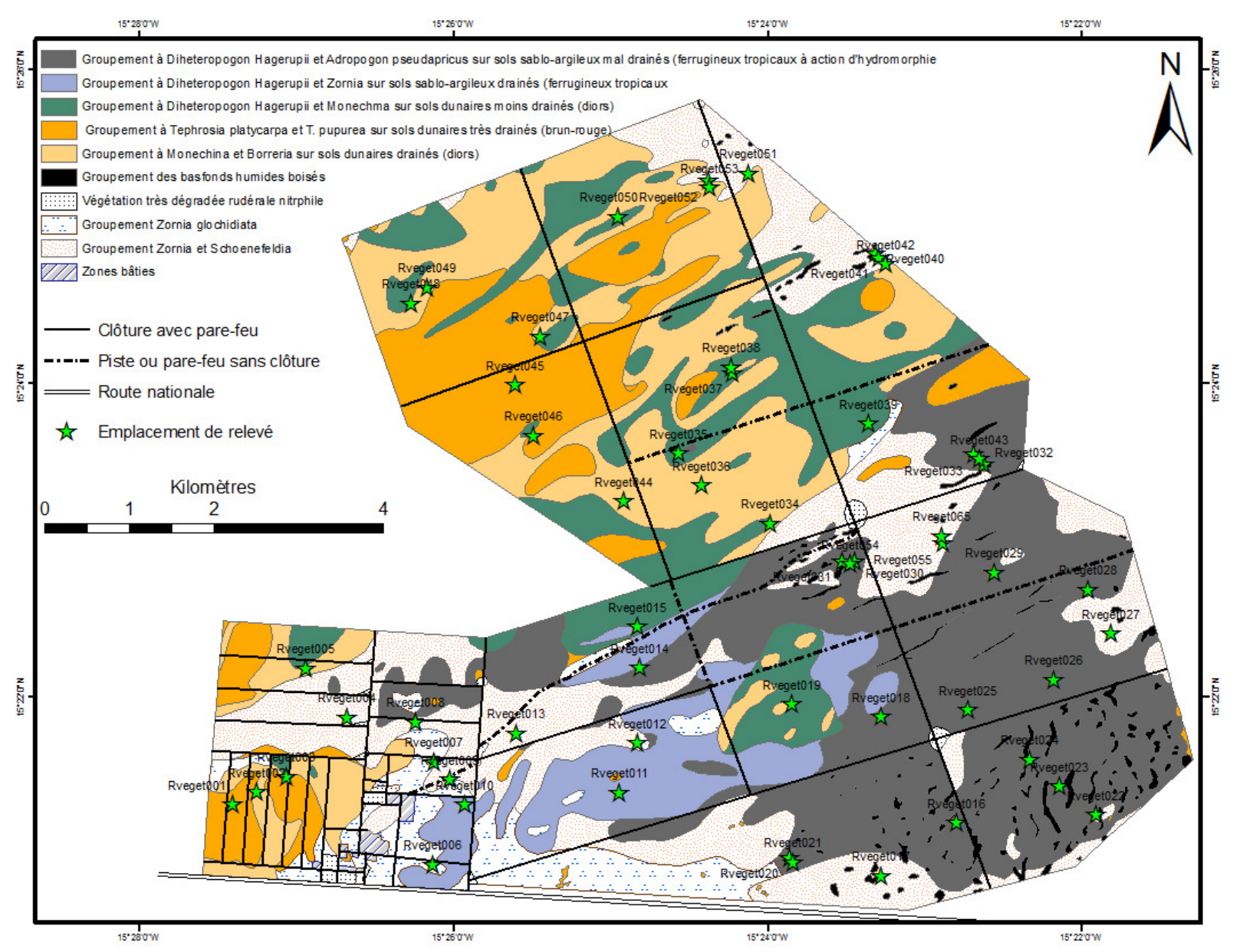

Figure 2 : carte de la végétation du CRZ de Dahra associée aux relevés effectués 
L'importance et la distribution des espèces dans les différents biotopes ont été étudiées en utilisant l'histogramme des classes de fréquences de Raunkiaer (1905) cité par Mangara et al., 2008, les indices de Shannon ( $H^{\prime}$ et $\left.E\right)$, et de Jaccard, qui utilisent la fréquence de présence. II en est de même que la fréquence absolue ou spécifique d'une espèce $\mathrm{E}$ dans $\mathrm{N}$ relevés, traduit par le nombre de présences de l'espèce $E$ dans les $N$ relevés. La fréquence relative d'une espèce $E$ dans $N$ relevés, exprimée en pourcentage est le rapport du nombre de présences de l'espèce $E$ sur l'ensemble des relevés, soit E/N (Mangara et al., 2008). Pour les deux années (1964 et 2011), les espèces recensées ont été regroupées en 5 classes de fréquence selon I'histogramme de Raunkiaer (1905) cité par Mangara et al., 2008 présenté au tableau 1.

Tableau 1 : Correspondance entre les classes de fréquence de Raunkiaer (1905) cité par Mangara et al., 2008 et les fréquences relatives.

\begin{tabular}{|cc|}
\hline Classes & Fréquences relatives (\%) \\
\hline I & 0 à 20 \\
\hline II & 21 à 40 \\
\hline III & 41 à 60 \\
\hline IV & 61 à 80 \\
\hline V & 81 à 100 \\
\hline
\end{tabular}

La contribution spécifique liée à la fréquence de chaque espèce ou CSi expression de la contribution apportée par une espèce dans une végétation donnée, à partir de sa fréquence absolue. Elle est calculée par la formule proposée par Daget et Poissonet (1969), à savoir :

$\mathrm{CSi}=\frac{F S(e)}{\sum_{1}^{n} F S}$

Où $F S(e)$ est la fréquence absolue de l'espèce $(e)$ et $\sum_{1}^{n} F S$, la somme des fréquences absolues de toutes les espèces recensées $(\mathrm{n})$.

L'indice de diversité de Shannon $\left(\mathrm{H}^{\prime}=-\Sigma \mathrm{fi} \log _{2} \mathrm{fi}\right.$, avec 0 $<\mathrm{fi}<1$ ), qui repose sur la théorie de l'information, suppose que la diversité dans un écosystème peut être mesurée comme l'information contenue dans un message ou un code. Plus l'indice est élevé, plus la diversité est grande. L'évaluation de l'équitabilité ( $E=$ $H^{\prime} / H \max$ ) est utile pour détecter les changements dans la

\section{RÉSULTATS}

Variation de la composition floristique: La composition floristique de CRZ a connu une baisse significative. Le nombre d'espèces présentes (herbacées et ligneux) qui était de 152 en 1964 est passé à 92 en 2011, soit une baisse de 60 espèces. Quatre-vingt-dix (90) des espèces recensées en 1964 sont « absentes " (dont 81 herbacées et 9 ligneux) en 2011, même si on a pu identifier 31 «nouvelles présences» (dont 27 structure d'une communauté et, ainsi, le niveau d'organisation de cette dernière. On l'appelle aussi régularité ; c'est le rapport entre la diversité observée $\left(\mathrm{H}^{\prime}\right)$ et la diversité maximale $\left(H \max =\log _{2} S\right)$, $S$ étant le nombre d'espèces. Les indices de Jacccard $(P \mathrm{Pj})=\mathrm{c} /$ $(a+b+c) \times 100$ où $: a=$ nombre d'espèces de la liste $a$ (relevé $A$ ) ; $b=$ nombre d'espèces de la liste $b$ (relevé $B$ ) ; $c=$ nombre d'espèces communes) permettent de quantifier la similarité entre habitats (ou milieux). La similarité augmente avec la valeur de ces indices (Akpo et Grouzis, 2004). La méthode de la récolte intégrale a été utilisée pour l'évaluation de la phytomasse herbacée. Trente (30) échantillons de phytomasse ont été prélevés sur une superficie d'environ d'un hectare à l'aide d'un carré d'un mètre de côté, sur chaque site. La phytomasse moyenne du couvert herbacé du centre a été calculée pour ces deux périodes et leurs confrontations ont permis d'avoir une idée sur la situation de sa production herbagère. Une ANOVA est faite pour la phytomasse au seuil de $5 \%$ (test de Tukey).

herbacées et 4 ligneux) au cours de cette dernière année de suivi. Le nombre de genre est passé de 94 à 56 et celui de la famille de 41 à 30 (Tableau 2). Le couvert herbacé était riche de 129 espèces réparties en 78 genres et 30 familles en 1964 alors qu'en 2011 il ne présentait que 74 espèces réparties en 44 genres et 20 familles (Tableau 2). 


\section{Ndiaye et al. . J. Appl. Biosci. Étude comparée de la végétation de 1964 et 2011 en milieu pâturé : Cas du}

CRZ de Dahra

Tableau 2 : variation de la richesse floristique (herbacée et ligneuse) du CRZ de Dahra pour les années 1964 et 2011

\begin{tabular}{|cllcccc|}
\hline \multirow{2}{*}{ Années } & \multicolumn{1}{c}{$\begin{array}{c}\text { Type de } \\
\text { végétation }\end{array}$} & $\begin{array}{c}\text { Nombre } \\
\text { d'espèces } \\
\text { présentes }\end{array}$ & $\begin{array}{c}\text { Nombre } \\
\text { d'espèces } \\
\text { absentes }\end{array}$ & $\begin{array}{c}\text { Nombre de } \\
\text { genres }\end{array}$ & $\begin{array}{c}\text { Nombre de } \\
\text { familles }\end{array}$ \\
\hline \multirow{3}{*}{1964} & Herbacé & 129 & 27 & 78 & 30 \\
\cline { 2 - 6 } & Ligneux & 23 & 4 & 16 & 11 \\
\cline { 2 - 6 } & Total & 152 & 31 & 94 & 40 \\
\hline \multirow{3}{*}{2011} & Herbacé & 74 & 81 & 44 & 20 \\
\cline { 2 - 6 } & Ligneux & 18 & 9 & 12 & 30 \\
\cline { 2 - 7 } & Total & 92 & 90 & 56 & 30 \\
\hline
\end{tabular}

Les espèces herbacées étaient plus représentées en 1964 et 2011 dans la famille des Poaceae $(34,88 \%$ contre $29,73 \%)$, des Fabaceae (17,05\% contre 18,91\%), et des Rubiaceae $(6,20 \%$ contre $10,81 \%)$. Les Cyperaceae qui étaient bien représentées en terme d'espèces en $1964(6,98 \%)$ ont connu une baisse en $2011(1,35 \%)$ par contre les Convolvulaceae ont connu une hausse et sont passées de $4,65 \%$ à 10,81\% (tableau 3).

Tableau 3 : Variation de la fréquence des espèces par famille en 1964 et 2011 au niveau du CRZ de Dahra

\begin{tabular}{|c|c|c|c|c|c|}
\hline Familles & $\begin{array}{l}\text { fréquence } \\
\text { sp/fam } 1964 \\
\text { (\%) }\end{array}$ & $\begin{array}{l}\text { fréquence } \\
\text { sp/fam } 2011 \\
(\%)\end{array}$ & Familles & $\begin{array}{l}\text { fréquence } \\
\text { sp/fam } 1964 \\
(\%)\end{array}$ & $\begin{array}{l}\text { fréquence } \\
\text { sp/fam } 2011 \\
(\%)\end{array}$ \\
\hline Acanthaceae & 3,10 & 5,41 & Malvaceae & 3,10 & 1,35 \\
\hline Aizoaceae & 2,33 & 4,05 & Menispermaceae & 0,78 & 0,00 \\
\hline Amaranthaceae & 2,33 & 1,35 & Onagraceae & 0,78 & 0,00 \\
\hline Asparagaceae & 1,55 & 0,00 & Orobanchaceae & 1,55 & 0,00 \\
\hline Asteraceae & 1,55 & 0,00 & Pedaliaceae & 1,55 & 2,70 \\
\hline Boraginaceae & 0,78 & 0,00 & Phyllanthaceae & 1,55 & 0,00 \\
\hline Burseraceae & 0,00 & 1,35 & Poaceae & 34,88 & 29,73 \\
\hline Caryophyllaceae & 0,78 & 1,35 & Polygalaceae & 0,78 & 0,00 \\
\hline Commelinaceae & 1,55 & 1,35 & Portulacaceae & 0,78 & 0,00 \\
\hline Convolvulaceae & 4,65 & 10,81 & Rubiaceae & 6,20 & 10,81 \\
\hline Cucurbitaceae & 4,65 & 2,70 & Solanaceae & 0,00 & 1,35 \\
\hline Cyperaceae & 6,98 & 1,35 & Sapindaceae & 0,78 & 1,33 \\
\hline Euphorbiaceae & 3,10 & 0,00 & Sphenocleaceae & 1,55 & 1,35 \\
\hline Fabaceae & 17,05 & 18,91 & Sterculiacea & 0,78 & 1,35 \\
\hline Geraniaceae & 0,78 & 0,00 & Tiliaceae & 0,00 & 1,35 \\
\hline Gisekiaceae & 0,78 & 0,00 & Tribullaceae & 0,78 & 1,35 \\
\hline Lamiaceae & 0,78 & 0,00 & & & \\
\hline
\end{tabular}

NB : sp=espèce ; fam= famille

S'agissant du ligneux, vingt et trois (23) espèces ont été dénombrées en 1964 réparties en 16 genres et 11 familles tandis qu'en 2011 le nombre d'espèces était de 18 réparti en 12 genres et 10 familles (tableau 2). Les espèces Euphorbia balsamica, Calotropis procera,
Leptadenia hastata et Ziziphus micronata étaient absentes en 1964 tandis qu'en 2011, le nombre d'espèces absentes, au nombre de 9 correspond à Acacia macrostachya, Bauhinia rufescens, Commiphora africana, Guiera senegalensis, Lannea acida, Piliostigma 
reticulatum, Sterculia setigera, Stereospermum kunthianum et Tamarindus indica. La famille des Mimosacées et celle des Combrétacées ont été les plus représentées en termes d'espèces au cours de ces deux années, avec des taux respectifs de 26,09 et $21,74 \%$ en 1964 puis de 27,78 et $22,22 \%$ en 2011 (figure 3 ).

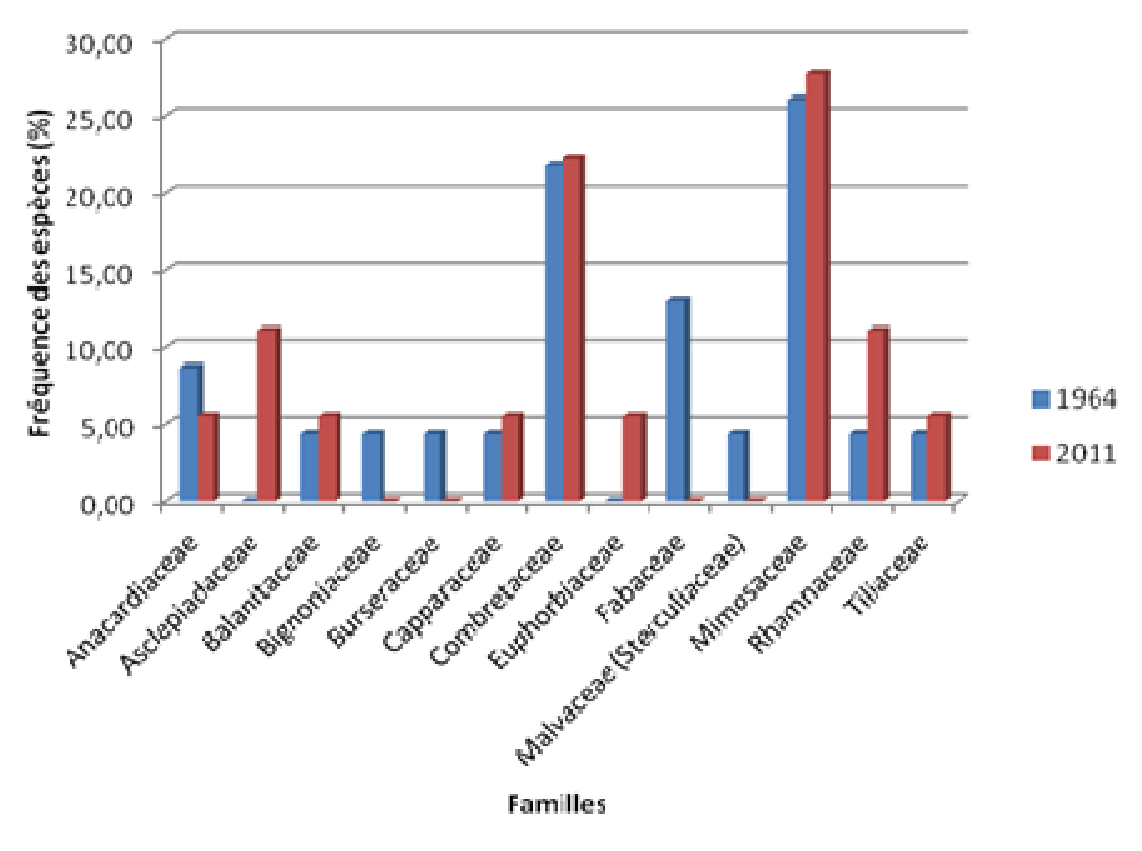

Figure 3 : Répartition des espèces ligneuses par familles botaniques en 1964 et 2011 au niveau du CRZ de Dahra

Variation de la fréquence et de la diversité floristique dans la station de Dahra : L'analyse des courbes de fréquences de 1964 et 2011 indique une prédominance des espèces accidentelles (I) et accessoires (II) et une faible présence des espèces de classe $V$ et IV dans la station du CRZ de Dahra pour ces deux périodes. Cette disposition en $L$ de l'histogramme de fréquence révèle une hétérogénéité et une instabilité du milieu (figure 4). Le nombre moyen d'espèces par relevé est de $31 \pm 8$ en 1964 avec $45,45 \%$ des relevés dépassant la moyenne spécifique et de $21 \pm 4$ espèces en 2011 avec $47,27 \%$ des relevés dépassant cette moyenne. La moyenne pour les deux périodes est de $26 \pm 8$ espèces dont $87,27 \%$ des relevés de 1964 qui présentait une richesse spécifique supérieure à cette moyenne alors qu'en 2011 il était de $9,09 \%$. Les espèces herbacées les plus fréquentes en
1964 étaient Andropogon amplectens divers.(87,50\%), Borreria stachydea $(87,50 \%)$, Zornia glochidiata (82,14\%), Cassia mimosoides (78,57\%), Merremia pinnata $(76,79 \%$ ) et Cenchrus biflorus (76,79\%) avec une contribution variant de 2,82 à 3,22\% alors qu'en 2011 les espèces Zornia glochidiata (94,55\%), Dactyloctenium aegyptium (90,91\%), Schoenfeldia gracilis $(90,91 \%)$, Alysicarpus ovalifolius (81,82\%), Chloris prieurii (76,36\%), Cenchrus biflorus (74,55\%) et Eragrostis tremula $(74,55 \%)$ étaient les plus représentées avec une contribution variant entre 4,56 et $57,8 \%$ (Tableau 4). La contribution spécifique des graminées est passée de 37 , $67 \%$ à $41,82 \%$, les légumineuses de $17,33 \%$ à $15,02 \%$ et les autres herbacés de 44,65 à $43,16 \%$ respectivement en 1964 et 2011. 
Data 1

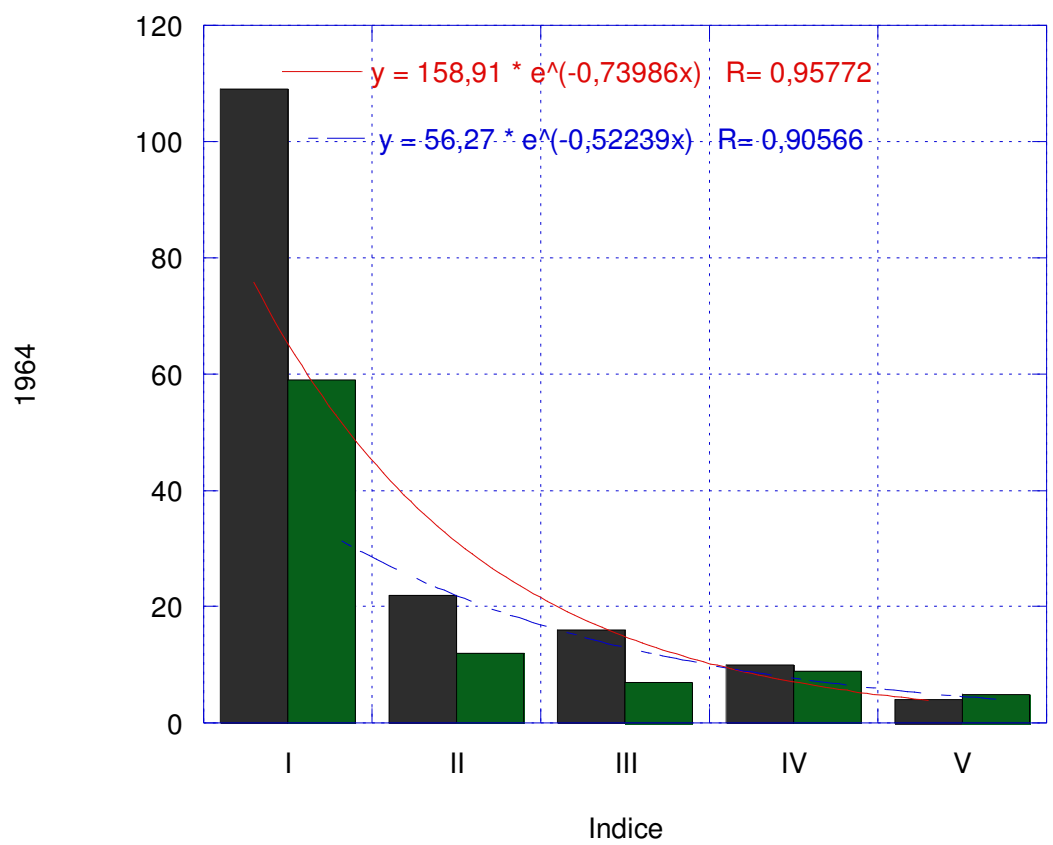

Figure 4 : Variation des histogrammes de fréquences entre 1964 et 2011

Tableau 4 : Fréquence et Contribution spécifique (CSi) de la flore herbacée du CRZ de Dahra de 1964 et 2011

\begin{tabular}{|c|c|c|c|c|c|c|c|}
\hline \multicolumn{3}{|c|}{1964} & & \multicolumn{4}{|c|}{2011} \\
\hline Espèces & $\begin{array}{l}\text { Fréquence } \\
\text { relative (\%) }\end{array}$ & $\begin{array}{l}\text { CSi } \\
(\%)\end{array}$ & IS & Espèces & $\begin{array}{l}\text { Fréquence } \\
\text { relative(\%) }\end{array}$ & $\begin{array}{l}\text { CSi } \\
(\%)\end{array}$ & $\begin{array}{c}\text { IS } \\
\mathrm{i}\end{array}$ \\
\hline $\begin{array}{l}\text { Andropogon amplectens } \\
\text { divers. }\end{array}$ & 87,50 & 3,22 & & Zornia glochidiata & 94,55 & 5,78 & 2 \\
\hline Borreria stachydea & 87,50 & 3,22 & 1 & $\begin{array}{l}\text { Dactyloctenium } \\
\text { aegyptium }\end{array}$ & 90,91 & 5,56 & 2 \\
\hline Zornia glochidiata & 82,14 & 3,02 & 2 & $\begin{array}{l}\text { Schoenefeldia } \\
\text { gracilis }\end{array}$ & 90,91 & 5,56 & 3 \\
\hline Cassia mimosoides & 78,57 & 2,89 & 2 & $\begin{array}{l}\text { Alysicarpus } \\
\text { ovalifolius }\end{array}$ & 81,82 & 5,01 & 2 \\
\hline Merremia pinnata & 76,79 & 2,82 & 2 & Chloris prieurii & 76,36 & 4,67 & 3 \\
\hline Cenchrus biflorus & 76,79 & 2,82 & 3 & Cenchrus biflorus & 74,55 & 4,56 & 3 \\
\hline Commelina forskalaei & 67,86 & 2,50 & 2 & Eragrostis tremula & 74,55 & 4,56 & 3 \\
\hline Digitaria adscendens & 67,86 & 2,50 & & Ipomea vagans & 70,91 & 4,34 & 2 \\
\hline Alysicarpus ovalifolius & 64,29 & 2,36 & 2 & Cassia tora (obs) & 69,09 & 4,23 & \\
\hline Polycarpaea linearifolia & 62,50 & 2,30 & 0 & Aristida mutabilis & 63,64 & 3,89 & 2 \\
\hline Borreria radiata & 62,50 & 2,30 & 0 & Achyrantes aspera & 63,64 & 3,89 & 1 \\
\hline $\begin{array}{l}\text { Dactyloctenium } \\
\text { aegyptium }\end{array}$ & 60,71 & 2,23 & 2 & Merremia pinnata & 60,00 & 3,67 & 2 \\
\hline
\end{tabular}


Ndiaye et al. . J. Appl. Biosci. Étude comparée de la végétation de 1964 et 2011 en milieu pâturé : Cas du CRZ de Dahra

\begin{tabular}{|c|c|c|c|c|c|c|c|}
\hline Eragrostis tremula & 58,93 & 2,17 & 3 & Merremia sp & 56,36 & 3,45 & \\
\hline Digitaria gayana & 53,57 & 1,97 & & Cynodon dactylon & 45,45 & 2,78 & \\
\hline Phyllanthus pentandrus & 51,79 & 1,90 & & $\begin{array}{l}\text { Merremia } \\
\text { aegyptium }\end{array}$ & 41,82 & 2,56 & 2 \\
\hline $\begin{array}{l}\text { Ceratotheca } \\
\text { sesamoides }\end{array}$ & 48,21 & 1,77 & 0 & Borreria linearis & 41,82 & 2,56 & \\
\hline Brachiaria xantholeuca & 48,21 & 1,77 & 3 & Corchorus tridens & 41,82 & 2,56 & 1 \\
\hline Sporobolus festivus & 48,21 & 1,77 & & Cucumis melo & 36,36 & 2,22 & 1 \\
\hline Ipomea pes-tigridis & 46,43 & 1,71 & 2 & $\begin{array}{l}\text { Ceratotheca } \\
\text { sesamoides }\end{array}$ & 34,55 & 2,11 & 0 \\
\hline Cenchrus prieurii & 46,43 & 1,71 & 3 & Digitaria gayana & 34,55 & 2,11 & \\
\hline Ipomea vagans & 44,64 & 1,64 & 2 & Tephrosia purpurea & 32,73 & 2,00 & \\
\hline Chloris pilosa & 44,64 & 1,64 & 3 & $\begin{array}{l}\text { Brachiaria } \\
\text { xantholeuca }\end{array}$ & 30,91 & 1,89 & 3 \\
\hline Chloris prieurii & 44,64 & 1,64 & 3 & & & & \\
\hline Cassia tora & 42,86 & 1,58 & & & & & \\
\hline Elionurus elegans & 42,86 & 1,58 & 1 & & & & \\
\hline Tephrosia linearis & 41,07 & 1,51 & & & & & \\
\hline Aristida mutabilis & 41,07 & 1,51 & 2 & & & & \\
\hline $\begin{array}{l}\text { Melothria } \\
\text { maderaspatana }\end{array}$ & 37,50 & 1,38 & & & & & \\
\hline $\begin{array}{l}\text { Pennisetum } \\
\text { pedicellatum }\end{array}$ & 37,50 & 1,38 & 3 & & & & \\
\hline Waltheria americana & 37,50 & 1,38 & & & & & \\
\hline Indigofera aspera & 33,93 & 1,25 & 2 & & & & \\
\hline Achyrantes argentea & 32,14 & 1,18 & 1 & & & & \\
\hline Brachiaria hagerupii & 32,14 & 1,18 & & & & & \\
\hline Tephrosia purpurea & 30,36 & 1,12 & & & & & \\
\hline Striga aspera & 30,36 & 1,12 & & & & & \\
\hline
\end{tabular}

NB : seules les espèces présentant une fréquence relative supérieure ou égale à $30 \%$ sont représentées avec leur contribution spécifique (CSi).

Les espèces Guiera senegalensis (82,14\%), Combretum glutinosum $(71,43 \%)$, Balanites aegyptiaca $(58,93 \%)$ et Acacia senegal (32,14\%) étaient les fréquentes dans le centre de Dahra en 1964 tandis qu'en 2011 c'était plutôt les espèces Balanites aegyptiaca $(87,27 \%)$, Leptadenia hastata $(76,26 \%)$, Acacia raddiana $(52,73 \%)$, Acacia seyal $(50,91 \%)$, Acacia nilotica $(38,18 \%)$ et Acacia senegal (23,64\%) (Tableau 5). Guiera senegalensis qui était majoritaire en 1964 a «disparu » au niveau du Centre de Dahra par contre Combretum glutinosum et Grewia bicolor ont fortement régressé. La contribution des espèces à la végétation ligneuse est passée de $44,64 \%$ à $4,75 \%$ pour la famille des Combretaceae, de 20,09 à 44,39\% pour les Mimosaceae et de 14,73 à $22,43 \%$ pour les Balanitaceae. 
Ndiaye et al. . J. Appl. Biosci. Étude comparée de la végétation de 1964 et 2011 en milieu pâturé : Cas du CRZ de Dahra

Tableau 5 : Fréquence et Contribution spécifique (CSi) de la flore ligneuse du CRZ de Dahra de 1964 et 2011

\begin{tabular}{|llrrrr|}
\hline & & \multicolumn{2}{c}{1964} & \multicolumn{2}{c|}{ 2011 } \\
\hline Familles & Espèces & FR (\%) & CSi (\%) & FR (\%) & CSi (\%) \\
\hline Mimosaceae & Acacia ataxacantha & 5,36 & 1,34 & 7,27 & 1,87 \\
\hline Mimosaceae & Acacia macrostachya & 1,79 & 0,45 & 0,00 & 0,00 \\
\hline Mimosaceae & Acacia nilotica & 12,50 & 3,13 & 38,18 & 9,81 \\
\hline Mimosaceae & Acacia raddiana & 3,57 & 0,89 & 52,73 & 13,55 \\
\hline Mimosaceae & Acacia senegal & 32,14 & 8,04 & 23,64 & 6,07 \\
\hline Mimosaceae & Acacia seyal & 25,00 & 6,25 & 50,91 & 13,08 \\
\hline Combretaceae & Anogeissis leiocarpus & 1,79 & 0,45 & 0,00 & 0,00 \\
\hline Balanitaceae & Balanites aegyptiaca & 58,93 & 14,73 & 87,27 & 22,43 \\
\hline Fabaceae & Bauhinia rufescens & 1,79 & 0,45 & 0,00 & 0,00 \\
\hline Capparaceae & Boscia senegalensis & 7,14 & 1,79 & 0,00 & 0,00 \\
\hline Asclepiadaceae & Calotropis procera & 0,00 & 0,00 & 21,82 & 5,61 \\
\hline Combretaceae & Combretum aculeatum & 10,71 & 2,68 & 7,27 & 1,87 \\
\hline Combretaceae & Combretum glutinosum & 71,43 & 17,86 & 3,64 & 0,93 \\
\hline Combretaceae & Combrteum micranthum & 12,50 & 3,13 & 7,27 & 1,87 \\
\hline Burseraceae & Commiphora africana & 5,36 & 1,34 & 0,00 & 0,00 \\
\hline Euphorbiaceae & Euphorbia balsamina & 0,00 & 0,00 & 1,82 & 0,47 \\
\hline Tiliaceae & Grewia bicolor & 28,57 & 7,14 & 0,00 & 0,00 \\
\hline Combretaceae & Guiera senegalensis & 82,14 & 20,54 & 0,00 & 0,00 \\
\hline Anacardiaceae & Lannea acida & 12,50 & 3,13 & 0,00 & 0,00 \\
\hline Asclepiadaceae & Leptadaena hastata & 0,00 & 0,00 & 76,36 & 19,63 \\
\hline Fabaceae & Piliostigma reticulatum & 1,79 & 0,45 & 0,00 & 0,00 \\
\hline Anacardiaceae & Sclerocarya birrea & 10,71 & 2,68 & 5,45 & 1,40 \\
\hline Malvaceae & Sterculia setigera & 3,57 & 0,89 & 0,00 & 0,00 \\
(Sterculiaceae) & Stereospermum & 1,79 & 0,45 & 0,00 & 0,00 \\
\hline Bignoniaceae & Kunthianum & 1,79 & 0,45 & 0,00 & 0,00 \\
\hline Fabaceae & 7,14 & 1,79 & 3,64 & 0,93 \\
\hline Ramnaceae & 0,00 & 0,00 & 1,82 & 0,47 \\
\hline Rhamnaceas & & & & \\
\hline & & & & \\
\hline
\end{tabular}

L'analyse des indices de diversité floristique montre que l'indice de diversité de Shannon (diversité a) et la régularité sont plus élevés en 1964 qu'en 2011, révélant une diversité plus importante pour la première année. La régularité nous indique une bonne représentation des espèces rencontrées dans la station pour les deux périodes confirmant l'hétérogénéité de cet écosystème (tableau 6). L'indice de similarité de Jaccard (diversité $\beta$ ) qui est de $19,51 \%$ révèle une faible similitude de la flore de la station du CRZ pour ces deux périodes. 


\section{Ndiaye et al. . J. Appl. Biosci. Étude comparée de la végétation de 1964 et 2011 en milieu pâturé : Cas du}

CRZ de Dahra

Tableau 6 : Paramètres de diversité de la station du CRZ de Dahra entre 1964 et 2011

\begin{tabular}{|lcc|}
\hline Paramètres de diversité & Année 1964 & Année 2011 \\
\hline Indice de Shannon (H') & 6,482 & 5,618 \\
\hline Régularité (E) & 0,877 & 0,8611 \\
\hline Indice de Jaccard (en \%) & \multicolumn{3}{|}{19,505} \\
\hline
\end{tabular}

\section{Variation de la phytomasse herbacée}

L'analyse statistique de la phytomasse de la station du CRZ, au cours des périodes 1964 et 2011, montre une baisse significative de la phytomasse de 2011 comparée à celle de 1964 avec des rendements respectifs de 0,85 0,48 T/ha et $1,71 \pm 0,64$ T/ha (figure 5).

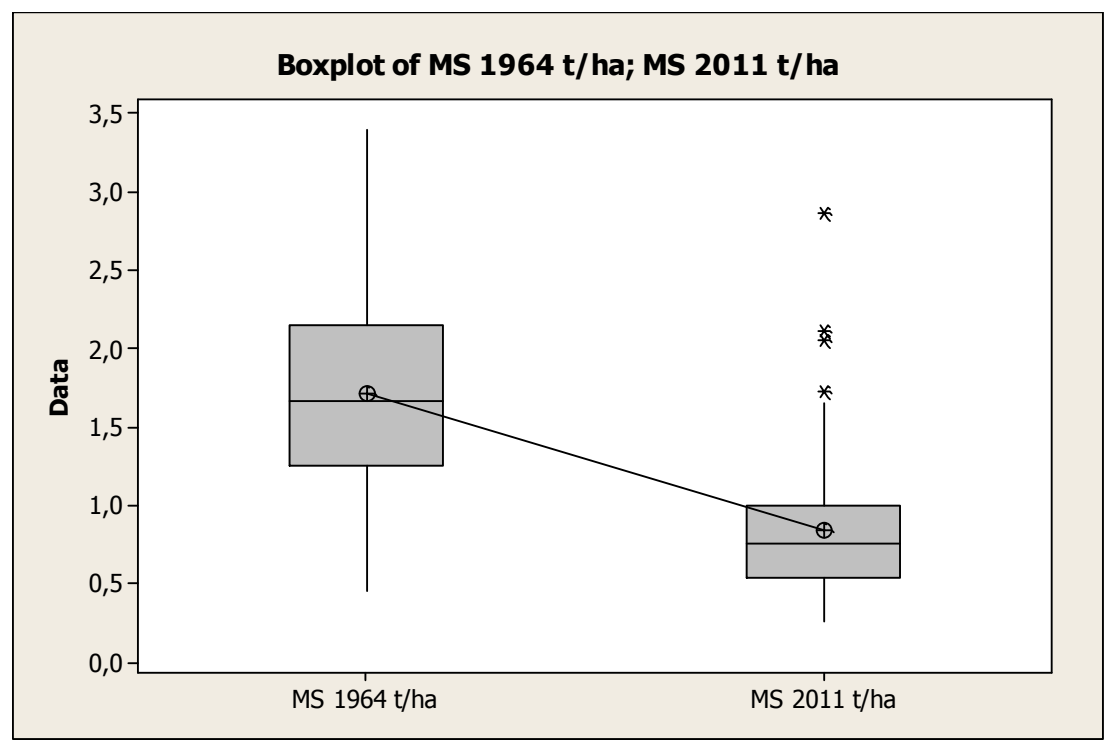

Figure 5 : Variation interannuelle de la phytomasse herbacée entre les périodes 1964 et 2011 au niveau de CRZ de Dahra

\section{DISCUSSION}

L'analyse de la composition et des paramètres de diversité floristique révèle une hétérogénéité de la végétation de la station du CRZ de Dahra pour les années 1964 et 2011. Cette situation est relatée par d'autres auteurs ayant travaillé dans les écosystèmes sahéliens qui l'imputent aux fluctuations climatiques associées aux activités anthropiques (Ndiaye, 2013, Diallo et al., 2011). Le couvert herbacé de Dahra était riche de 129 espèces réparties en 78 genres et 30 familles en 1964 alors qu'en 2011 il ne présentait que 74 espèces réparties en 44 genres et 20 familles. Ndiaye (2013) a recensé pour le Ferlo en 2012, 75 espèces réparties en 50 genres appartenant à 25 familles par contre Bakhoum (2013) indique une variation du nombre d'espèces de 72 à 14 et de celui des familles de 28 à 5 entre 1974 et 2010. Les espèces herbacées étaient plus représentées dans la famille des Poaceae et Fabaceae comme le confirment Bakhoum (2013) et Ndiaye (2013) dans leurs travaux. La diversité spécifique était plus importante en 1964 qu'en 2011, la similarité entre les biotopes pour ces deux périodes est faible $(19,5 \%)$ indiquant un changement important dans la composition floristique de la station. Ce dernier pourrait être attribué aux variabilités climatiques associées aux pressions zooanthropiques (Boudet, 1981, 1983; Hiernaux et le Houerou, 2006 ; Kluge, 1982 ; Knapp et Smith, 2001; Miehe, 1990 ; Ndiaye, 1981) et aux facteurs intrinsèques des végétaux (Grouzis, 1992; Grouzis et al., 1986 ; Ndiaye, 1981, 1995). Les espèces pérennes (Andropogon amplectens divers.) associées à des espèces à germination mixte (Zornia glochidiata) plus adaptées aux aléas climatiques et celle à germination lente (Borreria stachydea) présentaient les contributions spécifiques les plus importantes en 1964 alors qu'en 
2011, elles sont substituées par les espèces annuelles comme Alysicarpus ovalifolius, Schoenfeldia gracilis et Dactyloctenium aegyptium à germination moyenne à rapide et Zornia glochidiata, ces espèces sont plus résistantes au déficit hydrique (Grouzis, 1988). L'évolution du spectre floristique de la station de Dahra, caractérisée par la prédominance des herbacées annuelles et la raréfaction des herbacées pérennes localisées dans des endroits à situation hydrique favorable, indique des fluctuations pluviométriques importantes associées à des pressions de pâtures élevées. En effet, l'installation des espèces à germination rapide est révélatrice d'une situation pluviométrique favorable. Celle-ci est confirmée par les hausses pluviométriques notées entre 2009 et 2011. La présence des espèces à germination mixte (rapide ou lente) comme Zornia, qui est un faciès de dégradation qui a la particularité de coloniser les substrats dégradés (Boudet, 1983), est un indicateur du niveau de détérioration de ces parcours pouvant être imputé en partie par le surpâturage. Du point de vue qualitatif, les espèces les plus fréquentes répertoriées en 2011 constituent des espèces de bonne qualité fourragère car elles sont bien consommées en vert comme sous forme de paille sèche. Toutefois, Zornia glochidiata bien que appétée par le bétail, cause des problèmes de météorisation. Par contre en 1964, hormis Androgon amplectens divers. qui est de qualité moyenne, les autres espèces prédominantes étaient soit médiocres (Borreria stachydea) donc peu consommées par les ruminants, soit susceptibles de causer des problèmes sanitaires comme la météorisation (Zornia). La richesse floristique des ligneux a varié de 23 à 18 espèces entre les deux années (1964 et 2011). Ces résultats sont similaires à ceux de Bakhoum, 2013 réalisés dans un autre écosystème pastoral du Ferlo (Tessékéré) avec des effectifs variant de 23 à 17 espèces entre 1974 et 2010, mais diffèrent de ceux de Diouf, 2012 réalisé entre 1970 et 2000 à Thieul avec une richesse floristique variant de 18 à 8 espèces et de 31 à 19 espèces à Réwane. Ce couvert ligneux est caractérisé par la prédominance des Mimosacées, Balanitacées et les combrétacées qui renferment des espèces plus adaptées au déficit hydrique. Toutefois, la régression de certaines espèces zoo-anthrophiles comme Guiera senegalensis, Sclerocarya birrea, Combretum glutinosum et Grewia bicolor pourrait être expliquée par l'action combinée des conditions climatiques (Bakhoum, 2013 et
Ndiaye, 2013) et de leurs usages par les populations locales soit comme produits médicinaux ou matériaux de construction. Le développement de Calotropis procera est considéré par certains comme un indicateur de dégradation des parcours. Cette composante ligneuse est essentielle pour les pâturages sahéliens car elle apporte l'essentiel des éléments nutritifs (azote et protéine) dont a besoin le bétail en saison sèche (Baumer, 1992 ; Guinko et al., 1989 ; Guinko, 1991 ; Ickowicz et al., 2005 ; Von Maydell, 1983 et contribue également à l'amélioration de la diversité et de la phytomasse du couvert herbacée (Akpo et Grouzis, 2004). S'agissant de la phytomasse herbacée, la baisse constatée pourrait principalement être attribuée aux variations pluviométriques marquées par des années déficitaires importantes. Cette situation provoque la raréfaction d'espèces productrices (herbacées pérennes) au détriment des annuelles moins productrices. II s'y ajoute également d'autres facteurs secondaires comme la pression de pâture élevée de ces parcours causant des dommages importants dans les substrats (formation de croûtes, colmatage) entrainant une augmentation des plages nues qui limite le recouvrement du couvert herbacée et par la même occasion de la phytomasse herbacée. Le dynamisme de la composition et structure floristique, de la phytomasse herbacée des écosystèmes pastoraux sahéliens dépendent de plusieurs facteurs. Les facteurs climatiques, à travers la variabilité spatio-temporelle de la pluviométrie, le cumul et les évènements pluviométriques, constituent les éléments primordiaux dans le déterminisme de la composition botanique et de la phytomasse herbacée (Blair 1997; Boudet, 1981, 1983 ; Briggs et al., 1995 ; Hiernaux et le Houerou, 2006 ; Kluge, 1982 ; Knapp et Smith, 2001 ; Miehe, 1990 ; Tilman et Downing 1994 ; Ndiaye, 1981). II s'y ajoute des facteurs intrinsèques aux végétaux tels que la quantité et la qualité de la graine produite, les conditions de germination (Grouzis, 1992; Grouzis et al., 1986; Ndiaye, 1981, 1995), la vitesse de croissance et la physiologie de la plante (De Ridder et al., 1982 ; Sala et al., 1988 ; Grouzis, 1992 ; Huxman et al., 2004) ; les facteurs anthropiques tels que les activités pastorales (Carrière, 1996; Devineau, 1999 ; Gaucherand, 2005 ; Turner et al., 2005 ; Peco et al., 2006), agricoles et les feux de brousse provoqués et édaphiques (nature, structure, texture, situation hydrique et état de dégradation du sol).

\section{REMERCIEMENTS}

Nous remercions spécialement Animal Change pour le financement de cette activité, Ibrahima Mall et Dr Mouhamadou Sissokho pour leurs contributions. 


\section{BIBLIOGRAPHIE}

Akpo LE, Grouzis M, 2004. Interactions arbre/herbe en bioclimat semi-aride : influence de la pâture. Sécheresse 15 (3) : 253-61

Bakhoum A, 2013. Dynamique des ressources fourragères : indicateur de résilience des parcours communautaires de Tessékére au Ferlo, (Nord-Sénégal). Thèse de doctorat en Biologie, Productions et Pathologies Animales, option : Pastoralisme, École doctorale: Sciences de la Vie, de la Sante et de l'Environnement (ED-SEV)/UCAD. $115 p$

Baumer M, 1992. Trees as browse to support animal production. In A. Speedy et P.L. Pugliese (éds), Legume trees and other fodder trees as protein sources for livestock. Actes d'une consultation d'experts de la FAO, Kuala Lumpur, Malaisie, $p$. 1-10. FAO, Rome

Blair JM, 1997. Fire, $N$ availability, and plant response in grasslands: A test of the transient maxima hypothesis. Ecology 78: 2359-2368.

Boudet G, 1981. Systèmes de production d'élevage au Sénégal: étude du couvert herbacé (2ème campagne). ORSTOM-CNRF-LNERV-ORANAOCCGE-CERDAT-IEMVT-CTFT. 20p, annexes.

Boudet G, 1983. Systèmes de production d'élevage au Sénégal : étude du couvert herbacé (compte rendu de fin de campagne). ORSTOM-CNRFLNERV-ORANA-OCCGE-CERDAT-IEMVTCTFT. 27p, annexes.

Briggs JM, Knapp AK, 1995. Interannnual variability in primary production in Tallgrass prairie-Climate, soil-moisture, topographic position, and fires determinants of obvergrounds biomass. American journal of botany 82: 1024-1030.

Carrière M, 1996. Impacts des systèmes d'élevage pastoraux sur l'environnement en zones tropicales et sub-tropicales arides et sub-arides d'Afrique et d'Asie, CIRADEMVT, FAO, 80p.

Daget $\mathrm{Ph}$, Poissonet J, 1969. Analyse phytologique des prairies. CNRS-CEPE. Application agronomique Docum. 48 - $67 \mathrm{p}$.

De Ridder N, Stroosnijder L, Cissé AM, 1982. La productivité des pâturages sahéliens : une étude des sols, des végétations et de l'exploitation de cette ressource naturelle. Cours, PPS, Tome 1 , Théorie. 237p

Devineau JL, 1999. Rôle du bétail dans le cycle culturejachère en région soudanienne: la dissémination d'espèces végétales colonisatrices d'espaces ouverts (Bondoukuy, sud-ouest du Burkina
Faso). Revue d'écologie Terre et Vie, 54 : 97121.

Diallo, M, 1996. Study of the Senegalese pastures lands by the combined approach of the conventional method and geomatics. Ph.D. in Water Science, University of Quebec, Quebec.

Diallo A, Faye MN, Ndiaye O, Guissé A, 2011. Variations de la composition de la végétation herbacée des plantations de Acacia senegal (L.) Willd de la zone de Dahra (Ferlo).International Journal of Biological and Chemical Sciences 5 (3): 12501264.

Diouf JC, 2012. Dynamique du peuplement ligneux au Ferlo (Nord-Sénégal) : Conséquences et perspectives pour une gestion durables. Thèse 3ème cycle, FST. $262 \mathrm{p}$.

Gaucherand S, 2005. Effets des pratiques pastorales sur la biodiversité et sa dynamique dans les pelouses des alpages des alpes du nord : apport des traits fonctionnels des plantes. Thèse de doctorat de l'Ecole Nationale Supérieure Agronomique de Montpellier, École doctorale: Biologie Intégrative, Discipline: Biologie, 270p.

Dufrêne $M, 2003$. Méthodes d'analyse des données écologiques et biogéographiques. Version du 11/05/03. Adresse http://biodiversite.wallonie.be/outils/methodo/ home.html

Grouzis M, 1988. Structure, productivité et dynamique des systèmes écologiques sahéliens (Mare d'Oursi, Burkina Faso). Paris : éditions de I'ORSTOM, 336p.

Grouzis M, 1992. Germination et établissement des plantes annuelles sahéliennes. In : DidactiqueORSTOM, édition 1992. L'aridité une contrainte au développement. pp 267-282.

Grouzis M, Legrand E, Pale F, 1986. Germination des semences des régions semi-arides du sahel. Actes du Colloque "Les végétaux en milieu aride" : Tunisie (Jerba) : 534-552.

Guinko S, Zoungrana L, Zoungrana CY,1989. Étude des pâturages de la mare d'Oursi, Burkina Faso, rapport FAO, Ouagadougou, 51p.

Guinko S, 1991. Rôle des Acacia dans le développement rural au Burkina Faso et au Niger, Afrique de l'Ouest. Faculté des Sciences et Techniques, Université de Ouagadougou, Burkina Faso. P3751.

Hiernaux P, Le Houerou HN, 2006. Les parcours du Sahel. Sécheresse ; 17 (1-2) : 51-71. 
Huxman TE, Smith MD, Fay PA, Knapp AK, Shaw R, Loik M, Smith S, Tissue D, Zak J, Weltzin J, Pockman W, Sala O, Haddad B, Harte J, Koch GW, Schwinning S, Small E, Walliams DG, 2004. Response of net ecosystem gas exchange to a simulated precipitation pulse in a semi-arid grassland: the role of native versus non-native grasses and soil texture. Oecologia 141: 295-305.

Ickowicz A, Friot D, Guérin H, 2005. Acacia senegal, arbre fourrager sahélien ? Bois et forêts des tropiques, 2005, $\mathrm{N}^{\circ} 284$ (2) : 59-69.

Klug S, 1982. Inventaire et suivi (monitoring) de la végétation dans la parcelle d'élevage à Widou Thingoly (Ferlo-Sénégal). Rapport final d'enquêtes effectuées dans le cadre du Projet de l'Agence

Knapp AK, Smith MD, 2001. Interannual variability in net primary production and precipitation Response. Science, 293: U2-U2.

Mangara A, N'da Adopo AA, Boraud MKN, Kobenan K, Lejoly J, Traore D, 2008. Inventaire de la flore adventice en culture d'ananas (Ananas comosus (l.) Merr.) dans la localité de Bonoua en basse Côte d'Ivoire. Agronomie Africaine 20 (1) : 23 35.

Miehe S, 1990. Inventaire et suivi de la végétation dans les parcelles pastorales à Widou Thiengoly. Résultats de recherches effectuées de 1988 à 1990 et évaluation globale provisoire de l'essai de pâturage controlé après une période de 10 ans, GTZ, 108p.

Ndiaye 0,2013 . Caractéristiques des sols, de la flore et de la végétation du Ferlo, Sénégal. Thèse de doctorat en Écologie et Agroforesterie, EDSEV, UCAD-FST, 117p.

Ndiaye O, Diop AT, Akpo LE, Diène M, 2014. Dynamique de la teneur en carbone et en azote des sols dans les systèmes d'exploitation du Ferlo : cas du CRZ de Dahra. Journal of Applied Biosciences 83:7554- 7569 .

Ndiaye $P, 1981$. Contribution à l'étude du comportement des végétations herbacées annuelles. Thèse $3 \mathrm{e}$ cycle, univ. Dakar, 143 p. +3 annexes.

Ndiaye P, 1995. Le potentiel de reconstitution de la végétation herbacée au Sahel : réflexions sur le rôle des graines / The potential for reconstituting herbaceous vegetation in the Sahel : réflexions on the role of seeds. In: Revue de géographie de Lyon. Vol. 70 n 3-4, 1995. Sahel, la grande sécheresse. pp. 261-266.
Peco B, Sanchez A., Azcarate F., 2006. Abandonment in grazing systems: Consequences for vegetation and soil. Agriculture Ecosystems \& Environment, 113: 284-294.

Sala OE, Parton WJ, Joyce LA, Lauenroth WK, 1988. Primary Production of the Central grassland Region of the United States. Ecology, 69: 40-45.

Scoones I., 1999. Nouvelles orientations du développement pastoral en Afique : vivre dans un environnement incertain. (eds) Karthala-CTA. $362 p$

Sow RS, Denis JP, Trail JCM, Thiongane PI, Mbaye M, Diallo I, 1988. Productivité du zébu Gobra au centre de recherches zootechnique de Dahra (Sénégal). ISRA, 46p.

Tilman D, Downing JA, 1994. Biodiversity and Stability in Grasslands. Nature, 367:363-365.

Von Maydell HJ, 1983. Arbres et arbustes du Sahel. Leurs caractéristiques et leurs utilisations. Eschborn, Allemagne, Gtz, 531 p. 